
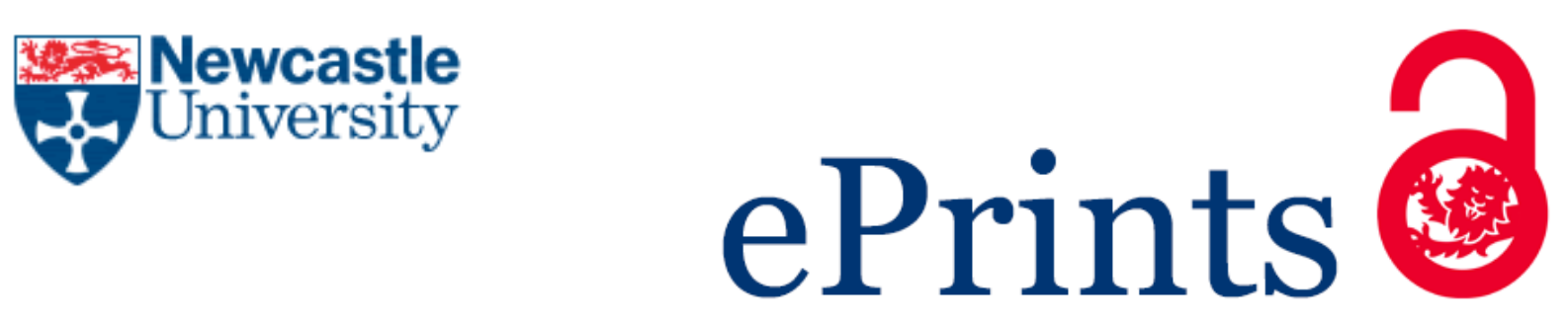

Birch-Machin MA, Bowman A.

Oxidative stress and ageing.

British Journal of Dermatology 2016, 175(Suppl. 2), 26-29.

\title{
Copyright:
}

This is the peer reviewed version of the following article: Birch-Machin MA, Bowman A. Oxidative stress and ageing. British Journal of Dermatology 2016, 175(Suppl. 2), 26-29, which has been published in final form at http://dx.doi.org/10.1111/bjd.14906 This article may be used for non-commercial purposes in accordance with Wiley Terms and Conditions for Self-Archiving.

DOI link to article:

http://dx.doi.org/10.1111/bjd.14906

Date deposited:

$02 / 12 / 2016$

Embargo release date:

26 September 2017 


\section{Oxidative stress and ageing}

\section{Prof. Mark A Birch-Machin and Bowman A}

Dermatological Sciences, Institute of Cellular Medicine, Newcastle University (medical school), Newcastle upon Tyne, NE24HH, UK, e-mail: mark.birch-machin@ncl.ac.uk

\section{Summary}

Oxidative stress is the resultant damage due to redox imbalances (increase in destructive free radicals (ROS) and reduction in antioxidant protection/pathways) and is linked to ageing in many tissues including skin. In ageing skin there are bioenergetic differences between keratinocytes and fibroblasts which provide a potential ageing biomarker. The differences in skin bioenergy are part of the mitochondrial theory of ageing which remains one of the most widely accepted ageing theories describing subsequent increasing free radical generation. Mitochondria are the major source of cellular oxidative stress and form part of the vicious cycle theory of ageing. External and internal sources of oxidative stress include UVR/IR, pollution (environment), lifestyle (exercise and diet), alcohol and smoking all of which may potentially impact on skin although many exogenous actives and endogenous antioxidant defence systems have been described to help abrogate the increased stress. This also links to differences in skin cell types in terms of the UVR action spectrum for nuclear and mitochondrial DNA damage (the latter a previously described UVR biomarker in skin). Recent work associates bioenergy production and oxidative stress with pigment production thereby providing another additional potential avenue for targeted anti-ageing intervention in skin.. This new data supporting the detrimental effects of the numerous wavelengths of UVR may aid in the development of cosmetic/sunscreen design to reduce the effects of photoageing. Recently, complex II of the mitochondrial electron transport chain appears to be more important than previously thought in the generation of free radicals (suggested predominantly by non-human studies). We investigated the relationship between complex II and ageing using human skin as a model tissue. The rate of complex II activity per unit of mitochondria was determined in fibroblasts and keratinocytes cultured from skin covering a wide age range. Complex II activity significantly decreased with age in fibroblasts $(P=0.015)$, but not in keratinocytes. This was associated with a significant decline in transcript expression ( $P=0.008$ and $P=0.001)$ and protein levels $(P=0.0006$ and $P=0.005)$ of the SDHA and SDHB catalytic subunits of complex II respectively. In addition there was a significant decrease in complex II activity with age $(P=0.029)$ that was specific to senescent skin cells, our study being the first to investigate these differences with senescence and skin age. There was no decrease in complex IV activity with increasing age, suggesting possible locality to complex II. Our study provides a future potential biomarker for monitoring the progression of skin ageing.

\section{The Mitochondrial Free Radical Theory of Ageing and Senescence}

Ageing describes the progressive functional decline of an organism over time, leading to an increased susceptibility to age-related diseases such as cancers, and eventually to the death of the organism. Despite a vast repertoire of ageing studies performed over the past century, the exact causes of ageing remain unknown. There are several theories of ageing that have been proposed 
including the 'wear and tear theory of ageing', the 'antagonistic pleiotropy theory of ageing' and the 'disposable soma theory of ageing'. There are a large number of studies into how and why we age that involve the mitochondria which are dynamic organelles found within the cytoplasm of eukaryotic cells, and are responsible for the production of the majority (approximately 90\%) of cellular energy via oxidative phosphorylation and the electron transport chain (ETC), in the form of adenosine triphosphate (ATP) [Birch-Machin 2006]. A natural by-product of oxygen metabolism in the mitochondria is the formation of reactive oxygen species (ROS). Free radicals, in particular ROS, contribute to oxidative stress through a variety of mechanisms. Interaction with nucleic acids (both in the mitochondria (mtDNA) and nucleus (nDNA), results in mutations that predispose to DNA strand breaks. Oxidation of lipids by ROS can damage cellular structures, for example the phospholipid cellular membranes, and result in premature cell death. However at low levels, ROS have important roles in cell signalling and homeostasis. Unlike nuclear DNA (nDNA), multiple copies of mitochondrial DNA (mtDNA) exist within each cell and are found in close proximity to the electron transport chain (ETC) making them extremely vulnerable to the effects of oxidative stress, exacerbated further by the fact that mtDNA has limited repair mechanisms [Anderson et al., 2014]. As mtDNA encodes subunits of the ETC, ROS-induced damage has the potential to alter ETC function and decrease the efficiency of ATP production. Harman proposed the 'free radical theory of ageing', which suggested that free radicals can affect and are possibly the cause of the ageing process of animals. [Barja 2013]. Within this theory the Vicious Cycle Theory of Ageing was included which proposes that ROS production from the mitochondrial ETC is able to cause damage to the mIDNA found in close proximity. MtDNA encodes the majority of mitochondrial proteins, so errors in gene expression of mtDNA may result in dysfunctional mitochondrial subunits. Dysfunctional mitochondria are then thought to contribute to further ROS leakage due to their inefficiency, which could then exacerbate mtDNA damage in a continuing vicious cycle [Bandy and Davison 1990, Kandola et al., 2015]. Evidence does exist in support of the theory in the various mechanisms and components involved in mitochondrial-related ageing [Hayakawa, 1992; Hudson et al., 1998; Kujoth et al., 2005; Trifonovic et al., 2005; Thayer et al., 2003; Birket et al., 2007]. More recently the process of mitohormesis has been proposed based on observations that low concentrations of ROS can induce a positive response whilst higher concentrations of ROS promotes damage; an interesting concept that different levels of oxidative stress may have opposite biological outcomes [Hudson et al., 2016].

Senescence describes the transformation of cells from a proliferating to a non-proliferating state, as a tumour suppressive mechanism to prevent cells with potentially cancerous DNA mutations from undergoing replication. Cells lose the ability to divide yet remain viable which is in contrast to biological ageing that describes the functional decline of a whole organism over time eventually leading to death [Bowman and Birch-Machin, 2016]. Mitochondrial dysfunction is thought to play a role in the increased levels of senescent cells observed with age [Passos et al., 2007; Birket et al., 2009; Passos et al., 2010] . However, not all cells in older organisms become senescent, as the induction of senescence is a stress response which only occurs in a minority of cells exposed to unfavourable conditions, or with mutations leading to oncogenic activation. More recently complex II has been shown to be implicated in human dermal fibroblast senescence and ageing [Anderson et al., 2014; Bowman and Birch-Machin, 2016; Boulton and Birch-Machin 2015]. Bowman et al demonstrated that complex II activity, transcript and protein levels decrease with age. Moreover, 
these effects are only seen in senescent cell populations. This result was shown to be specific to complex II as no change with complex IV was observed. Therefore this decrease in complex II activity could result in an increase in ROS resulting in mtDNA damage and oxidative stress which are known to contribute to the ageing process [Bowman and Birch-Machin 2016]. Recently, Correia-Melo et al. (2016) induced mitochondrial degradation to completely eliminate all mitochondria and demonstrated that these cells did not undergo hallmark changes of cellular.

\section{Sources of ROS}

The skin can undergo two distinct types of ageing; intrinsic (chronological) and extrinsic ageing (photoageing) via environmental insults, such as ultra violet radiation (UVR) [Birket and BirchMachin 2007; Krutman and Schroeder 2009]. Solar radiation is comprised of UVR (UVA and UVB), visible light and infrared (IR) [Krutman and Schroeder 2009] with a relative contribution of approximately $6 \%, 40 \%$ and $54 \%$ respectively. UVR is the main extrinsic influence of skin ageing [Kandola et al., 2015; Oyewole et al., 2013]; either by the production of ROS or via direct DNA damage. A possible mechanism as to how UVR is able to accelerate the ageing process could be via its interaction with mitochondria, where it may contribute to a vicious cycle of increasing damage [Kandola et al., 2015; Oyewole et al., 2014]. UVR may increase ROS levels, or cause mtDNA damage directly, which could result in an increase in mitochondrial dysfunction and a further production of ROS in a continuing vicious cycle and a putative increase in photoageing, as mitochondria are thought to play a prominent role in this process [Kandola et al., 2015]. This has led to the development of mtDNA as an established biomarker of UVR-induced damage [Birch-Machin et al., 2013].

Relatively little is known about the role of IRR in photoageing and/or mitochondria damage.

Evidence exists that IRR has a detrimental effect on human skin such as increased intracellular ROS, gene expression of MMPs[Hudson et al., 2016]. Interestingly, the mitochondria is believed to be a key cellular target involved in the pathogenesis of IRR induced premature ageing in the skin via the enzyme cytochrome C Oxidase (also known as complex IV of the ETC) acting as a chromophore for IRA.

Pollution is another major environmental cause of ROS production [Koohgoli et al., 2016]. Particles of less than $0.1 \mu \mathrm{m}$ diameter, defined as ultrafine particles, are particularly harmful with vehicle exhaust emissions being a major source [Li et al., 2003; Lodovici and Bigagli 2011]. Ultrafine particles specifically are able to penetrate tissues more easily and have been found to localise in mitochondria within epithelial and macrophage cells [Li et al., 2003] and most environmental pollutant agents have the ability to induce ROS [Lodovici and Bigagli 2011; Valavanidis 2009]. Adsorption at the surface of ultrafine particles leads to ROS generation which then induces oxidative stress; ultrafine particles that adsorb transition metal ions on their surface are able to produce ROS via the Fenton reaction described below [Lodovici and Bigagli 2011]. Whilst further work is required to support the relationship between pollution and premature ageing via mitochondria-mediated mechanisms, it 
provides important evidence to enhance knowledge on the role mitochondria play in the ageing process in both skin and other organs exposed to environmental pollutants such as lungs [Meyer 2013; Hou et al., 2010].

Mitochondria are responsible for the production of the majority of ROS within a cell, and these potentially harmful radicals are modulated by the expression of endogenous antioxidants involving the nuclear factor erythroid 2-related factor 2 (Nrf2)- kelch-like ECH-associated protein 1 (Keap1) pathway [Magesh et al., 2012] or via external anti-oxidants in the diet [Birch-Machin and Oyewole 2015; Darvin et al., 2008]. Carotenoid substances, found in fruit and vegetables, as well as vitamins $A, C$, and $E$ are said to be most protective and correlate negatively with levels of oxidative stress. Inducers of ROS, for example smoking and UVR exposure, can reduce levels of carotenoid antioxidants and thereby further potentiate levels of oxidative stress [Darvin et al., 2008]. In addition, a study showed that tomato paste can protect human skin against UVR-induced effects partially mediated by oxidative stress such as erythema (skin redness) and extracellular changes such as mtDNA damage [Rizwan et al., 2011].

In conclusion, the Mitochondrial Free Radical Theory of Ageing is one of the most accepted theories as to why we age, and because many age-related diseases appear to be linked to oxidative stress, there have been many attempts to modulate longevity using exogenous antioxidants from plant and food sources. Since ROS are important signalling molecules [Desler et al., 2011; Fischer et al., 2012], the dose and target of the administered antioxidant would have to be considered to prevent the alteration of cellular homeostasis. More recent developments in the field of mitohormesis provides an interesting concept for the varying roles oxidative stress plays in ageing. Continuing evidence supports the proposal that mitochondria are heavily implicated in the ageing process and warrants further work in this intriguing area.

\section{$\underline{\text { References }}$}

Anderson, A., et al., A role for human mitochondrial complex II in the production of reactive oxygen species in human skin. Redox Biology, 2014. 2: p. 1016-1022.

Bandy, B. and A.J. Davison, Mitochondrial mutations may increase oxidative stress: Implications for carcinogenesis and aging? Free Radical Biology and Medicine, 1990. 8(6): p. 523-539

Barja, G., Updating the Mitochondrial Free Radical Theory of Aging: An Integrated View, Key Aspects, and Confounding Concepts. Antioxidants \& Redox Signaling, 2013. 19(12): p. 1420-1445.

Birch-Machin, M.A., The role of mitochondria in ageing and carcinogenesis. Clinical and Experimental Dermatology, 2006. 31(4): p. 548-552.

Birch-Machin MA and Oyewole A. Mitochondrial-targeted antioxidants. FASEB 2015 Aug 7. pii: fj.15275404. 
Birch-Machin, M.A., E.V. Russell, and J.A. Latimer, Mitochondrial DNA damage as a biomarker for ultraviolet radiation exposure and oxidative stress. British Journal of Dermatology, 2013. 169: p. 914.

Birket, M.J. and M.A. Birch-Machin, Ultraviolet radiation exposure accelerates the accumulation of the aging-dependent T414G mitochondrial DNA mutation in human skin. Aging Cell, 2007. 6(4): p. 557-564

Birket, M.J., et al., The relationship between the aging-and photo-dependent T414G mitochondrial DNA mutation with cellular senescence and reactive oxygen species production in cultured skin fibroblasts. Journal of Investigative Dermatology, 2009. 129(6): p. 1361-1366

Bowman A and Birch-Machin MA The age-dependent decrease of mitochondrial complex II activity in human skin fibroblasts. J Invest Dermatol 2016 136, 912-919; doi:10.1016/j.jid.2016.01.017.

Boulton, S.J. and M.A. Birch-Machin, Impact of hyperpigmentation on superoxide flux and melanoma cell metabolism at mitochondrial complex II. FASEB Journal, 2015. 29(1): p. 346-353.

Correia-Melo, C., et al., Mitochondria are required for pro-ageing features of the senescent phenotype. The EMBO Journal, 2016.

Darvin, M.E., Patzelt, A., Knorr, F., Blume-Peytavi, U., Sterry, W. and Lademann, J. One-year study on the variation of carotenoid antioxidant substances in living human skin: influence of dietary supplementation and stress factors. Journal of biomedical optics. 13(4):044028 (2008).

Desler, C., Marcker, M.L., Singh, K.K. and Rasmussen, L.J. The importance of mitochondrial DNA in aging and cancer. J Aging Res. 2011:407536 (2011).

Fischer, F., Hamann, A. and Osiewacz, H.D. Mitochondrial quality control: an integrated network of pathways. Trends Biochem Sci. 37(7):284-92 (2012).

Hayakawa, M., et al., Age-associated oxygen damage and mutations in mitochondrial DNA in human hearts. Biochemical and Biophysical Research Communications, 1992. 189(2): p. 979-985.

Hou, L., et al., Airborne particulate matter and mitochondrial damage: a cross-sectional study. Environmental Health, 2010. 9(1): p. 1-9.

Hudson, E.K., et al., Age-associated change in mitochondrial DNA damage. Free Radical Research, 1998. 29(6): p. 573-579.

Hudson L, Bowman A, Rashdan E and Birch-Machin MA. Mitochondrial damage and ageing using skin as a model organ. Maturitas 2016 May 7. pii: S0378-5122(16)30100-1. doi:

10.1016/j.maturitas.2016.04.021

Kandola, K., A. Bowman, and M.A. Birch-Machin, Oxidative stress - A key emerging impact factor in health, ageing, lifestyle and aesthetics. International Journal of Cosmetic Science, 2015. 37: p. 1-8.

Koohgoli, L.H., Simon Wilkinson, Bhaven Chavan and Mark A Birch-Machin, Bad air gets under your skin. Experimental Dermatology, 2016 in press 
Krutmann, J. and P. Schroeder, Role of mitochondria in photoaging of human skin: The defective powerhouse model. Journal of Investigative Dermatology Symposium Proceedings, 2009. 14(1): p. 44-49.

Kujoth, C.C., et al., Medicine: Mitochondrial DNA mutations, oxidative stress, and apoptosis in mammalian aging. Science, 2005. 309(5733): p. 481-484.

Li, N., Sioutas, C., Cho, A., Schmitz, D., Misra, C., Sempf, J., et al. Ultrafine particulate pollutants induce oxidative stress and mitochondrial damage. Environmental health perspectives. 111(4):45560 (2003).

Lodovici, M. and Bigagli, E. Oxidative Stress and Air Pollution Exposure. Journal of Toxicology. 2011:487074 (2011).

Magesh, S., Chen, Y. and Hu, L. Small molecule modulators of Keap1-Nrf2-ARE pathway as potential preventive and therapeutic agents. Medicinal research reviews. 32(4):687-726 (2012).

Meyer, J.N., et al., Mitochondria as a Target of Environmental Toxicants. Toxicological Sciences, 2013.

Oyewole, A.O., et al., Comparing the effects of mitochondrial targeted and localized antioxidants with cellular antioxidants in human skin cells exposed to UVA and hydrogen peroxide. The FASEB Journal, 2014. 28(1): p. 485-494.

Passos, J.F., et al., Mitochondrial dysfunction accounts for the stochastic heterogeneity in telomeredependent senescence. PLoS Biology, 2007. 5(5): p. 1138-1151.

Passos, J.F., et al., Feedback between $p 21$ and reactive oxygen production is necessary for cell senescence. Molecular Systems Biology, 2010. 6.

Rizwan M; Rodriguez-Blanco I; Harbottle A; Birch-Machin MA, Watson REB and Rhodes LE. Tomato paste rich in lycopene protects against cutaneous photodamage in humans in vivo. Brit J Dermatol 2011, 164, 154-162.

Roxanna Koohgoli, L.H., Simon Wilkinson, Bhaven Chavan and Mark A Birch-Machin, Bad air gets under your skin. Experimental Dermatology, 2016 in press.

Thayer, R.E., et al., A maternal line study investigating the 4977-bp mitochondrial DNA deletion. Experimental Gerontology, 2003. 38(5): p. 567-571.

Trifunovic, A., et al., Somatic mtDNA mutations cause aging phenotypes without affecting reactive oxygen species production. Proceedings of the National Academy of Sciences of the United States of America, 2005. 102(50): p. 17993-17998.

Valavanidis, A., Vlachogianni, T. and Fiotakis, K. Tobacco smoke: involvement of reactive oxygen species and stable free radicals in mechanisms of oxidative damage, carcinogenesis and synergistic effects with other respirable particles. International journal of environmental research and public health. 6(2):445-62 (2009). 